\title{
Development of Animal Models of Otitis Media
}

\author{
Moo Kyun Park and Byung Don Lee \\ Department of Otolaryngology-Head and Neck Surgery, Soonchunhyang University College of Medicine, Seoul, Korea
}

\author{
Received February 20, 2013 \\ Revised February 28, 2013 \\ Accepted March 10, 2013 \\ Address for correspondence \\ Byung Don Lee, MD, PhD \\ Department of Otolaryngology-Head \\ and Neck Surgery, Soonchunhyang \\ University College of Medicine, \\ 59 Daesagwan-ro, Yongsan-gu, \\ Seoul 140-743, Korea \\ Tel $+82-2-709-9364$ \\ Fax $+82-2-709-9360$ \\ E-mail Bdlee12@schmc.ac.kr
}

\begin{abstract}
Otitis media is defined as inflammation of the middle ear, including the auditory ossicles and the Eustachian tube. Otitis media is a major health problem in many societies. The causes of otitis media includes infection and anatomic/physiologic, host, and environmental factors. In general, otitis media is a childhood disease, and anatomic and physiologic changes have great effects on its development. Thus, in vitro or human experimental studies of otitis media are difficult. Several experimental animal models have been introduced to investigate the pathogenesis and treatment of otitis media. However, none are ideal. The aim of this review is to provide a brief overview of the current status of animal models of otitis media with effusion, acute otitis media, and cholesteatoma. This review will assist determination of the most appropriate animal models of otitis media.

Korean J Audiol 2013;17:9-12
\end{abstract}

\section{Introduction}

Otitis media is one of the most common childhood diseases and is the most frequent reason for visits to a physician. ${ }^{1)}$ At least four-fifths of children will have experienced one or more episodes of otitis media by the age of 3 years. ${ }^{2}$ In addition, otitis media affects not only the child, but also the caregiver of the child. It affects hearing and balance, and eventually causes poor language development and poor educational performance. ${ }^{3,4)}$ The financial burden associated with otitis media in children 5 years of age and younger is estimated to be $\$ 5$ billion annually in the United States. ${ }^{5)}$

The causes of otitis media include infections and anatomic/physiologic, host, and environmental factors. ${ }^{4}$ From an anatomical viewpoint, Eustachian tube function is important for preventing the development of effusion in the middle ear. The Eustachian tube contributes to pressure regulation, protection, and clearance of the middle ear cavity. ${ }^{6}$ Cholesteatomas of the middle ear may also cause otitis media. ${ }^{7)}$ The causes of otitis media commonly overlap with one another. Recently, environmental factors such as smoking and air pollution have been studied in vitro and in vivo. ${ }^{8,9)}$ However, simulating these environmental factors in animal studies is problematic because the appropriate dosages and time frames are unknown.

Experimental otitis media is an active research field in otology. However, studies on humans are difficult in cases of new- ly developed treatments or drugs. In addition, studies on humans require the results of animal studies according to most research regulations. In vitro research is a good method of drug screening. It requires less time, and dose-response curves are easy to obtain. However, in vitro studies cannot reflect the full range ofanatomical and physiological effects. Several models of otitis media effusion, acute otitis media, and cholesteatoma have been developed (Table 1). ${ }^{10)}$ We herein review the most recently developed animal models and describe our own experience with several of them.

\section{Animal Models of Otitis Media with Effusion}

There are two categories of animal models of otitis media. One is Eustachian tube ligation or cauterization using a transneck or trans-oral approach, ${ }^{11)}$ and the other is injection of chemical materials through the tympanic membrane. ${ }^{12)}$

Dysfunction of the Eustachian tube is an important anatomical cause of otitis media with effusion. To simulate this condition, one can block the Eustachian tube by ligation or cauterization. Unfortunately, this is irreversible. The transneck approach is an accurate method of blocking the tube. However, it is more time-consuming than the trans-oral approach. Briefly, a rat or other rodent is placed in the supine position after proper intraperitoneal analgesic injection. We pre- 
Table 1. Comparison of various animal models of otitis media

\begin{tabular}{|c|c|c|c|c|}
\hline Models & Difficulty & Time & Reproducibility & Other \\
\hline \multicolumn{5}{|l|}{ Otitis media with effusion } \\
\hline \multicolumn{5}{|l|}{ Ligation or cauterization } \\
\hline Trans-neck approach & Difficult & $30 \mathrm{~min}$ & High & \\
\hline Trans-oral approach & Moderate & $<5 \min$ & Low & \\
\hline Injection methods & Easy & $<5 \min$ & Moderate & \\
\hline Oxgrl knock-out mouse & Easy & & High & \\
\hline Acute otitis media & Easy & $<5 \min$ & Moderate & High mortality \\
\hline Inoculation via trans-tympanic membrane & & & & \\
\hline \multicolumn{5}{|l|}{ Cholesteatoma } \\
\hline External auditory canal ligation & Moderate & $<10 \min$ & High & Expensive \\
\hline Injection methods & Easy & $<5 \min$ & Moderate & \\
\hline
\end{tabular}
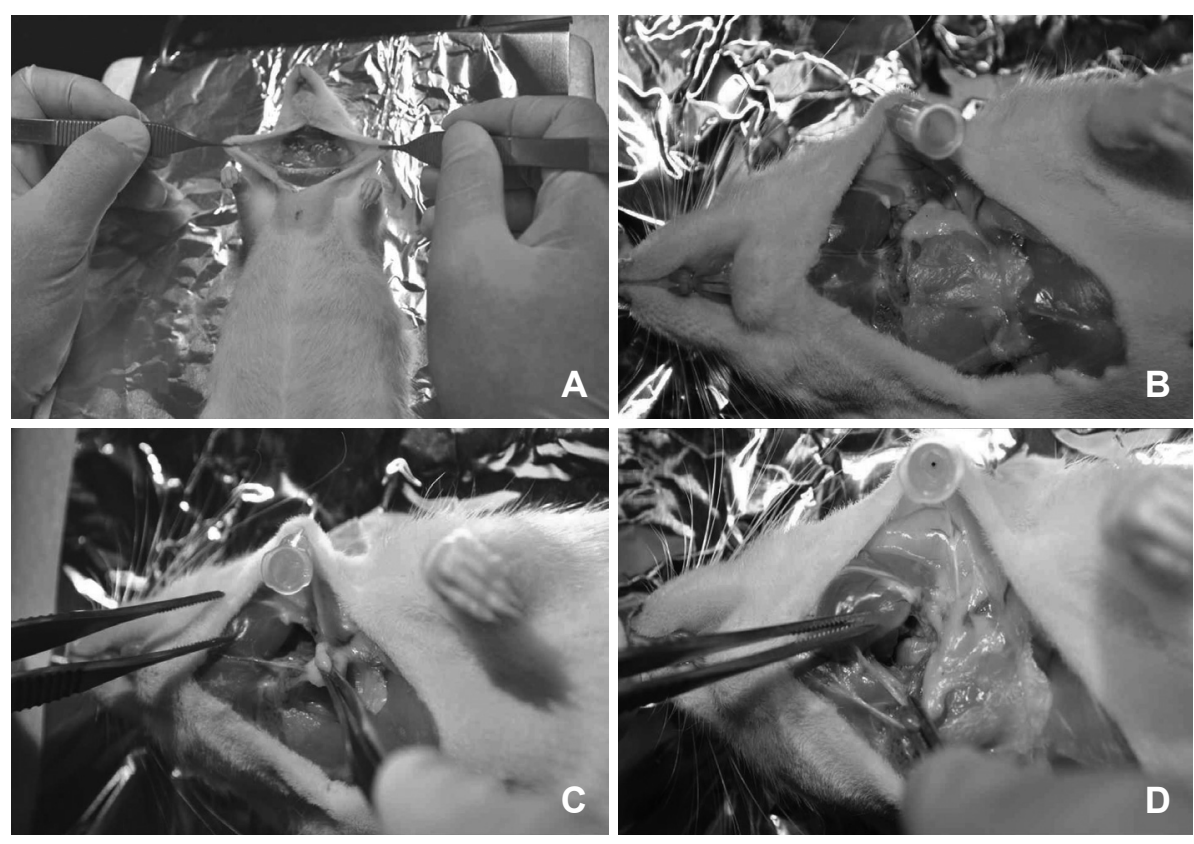

Fig. 1. Trans-neck approach to ligation or cauterization of the Eustachian tube for otitis media with effusion. A: Midline incision. B: Elevation of platysma. C: SCM and belly of digastric muscle. D: Exposure of bulla and Eustachian tube orifice. SCM: sternocleidomastoid muscle.

fer tiletamine/zolazepam (Zoletil ${ }^{\circledR} 50$; Virbac Lab, Carros, France) $(0.02 \mathrm{~mL} / 100 \mathrm{mg})$. A longitudinal midline incision is made at the neck, and the platysma muscle is elevated. After identifying the sternocleidomastoid muscle and belly of the digastric muscle, the facial nerve can be found at distal to the bulla. Arteries that are located medially should be controlled. Through meticulous dissection, the orifice of the Eustachian tube can be seen below the facial nerve and belly of the digastric muscle. Bleeding can be controlled with electrical cauterization (Fig. 1). The orifice of the Eustachian tube can be cauterized, the cartilage portion of the Eustachian tube can be ligated using electrical cautery or nylon, or the tube can be blocked using dental material after the bulla has been exposed. ${ }^{10,11,13)}$ Bacterial pathogens can be added to induce suppurative otitis media. ${ }^{11)}$

The trans-oral approach is a simpler method of blocking the Eustachian tube. ${ }^{14)}$ However, consistent results are not easy to obtain. After opening the mouth wide using a small blade or mouth gag, an electrical cautery tip is placed on the midline of the soft palate and directed laterally. The orifice of the Eustachian tube is located about $5 \mathrm{~mm}$ posterior to the junction of the hard and soft palates (Fig. 2). Severe thermal damage around the Eustachian tube can induce severe bleeding or poor oral intake. Some animals cannot bear this stress. Antibiotics can help to lower the mortality rate. Consistent results are not easy to obtain with this method because of the poor visual field.

When the soft palate is opened with a wide incision, the pharyngeal orifice of the Eustachian tube can be identified under microscopic visualization. This approach provides a better visual field. Trichloroacetic acid (50\%) can be used instead of electrical cautery. ${ }^{14)}$

Injection of chemical materials through the tympanic membrane is easier than the above-described methods. A spinal needle can be used. Histamine solution $(0.1 \mathrm{~mL})$ has been 

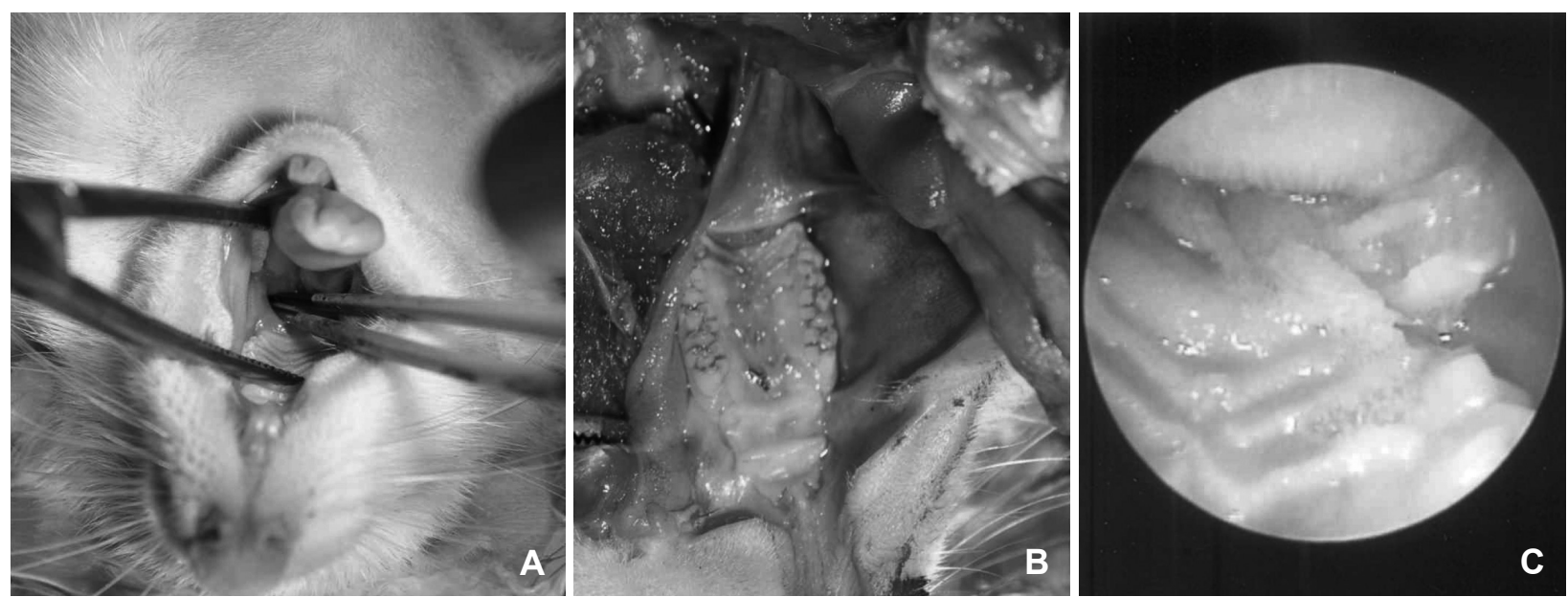

Fig. 2. Trans-oral approach to ligation or cauterization of the Eustachian tube for otitis media with effusion. A: Electrical cautery of pharyngeal orifice of the Eustachian tube. B: Pharyngeal incision and bulla exposure. C: Three days postoperatively.

Fig. 3. Result 4 days after induction of acute otitis media by inoculation of a bacterial pathogen via the trans-tympanic membrane. A: Right ear. B: Left ear.
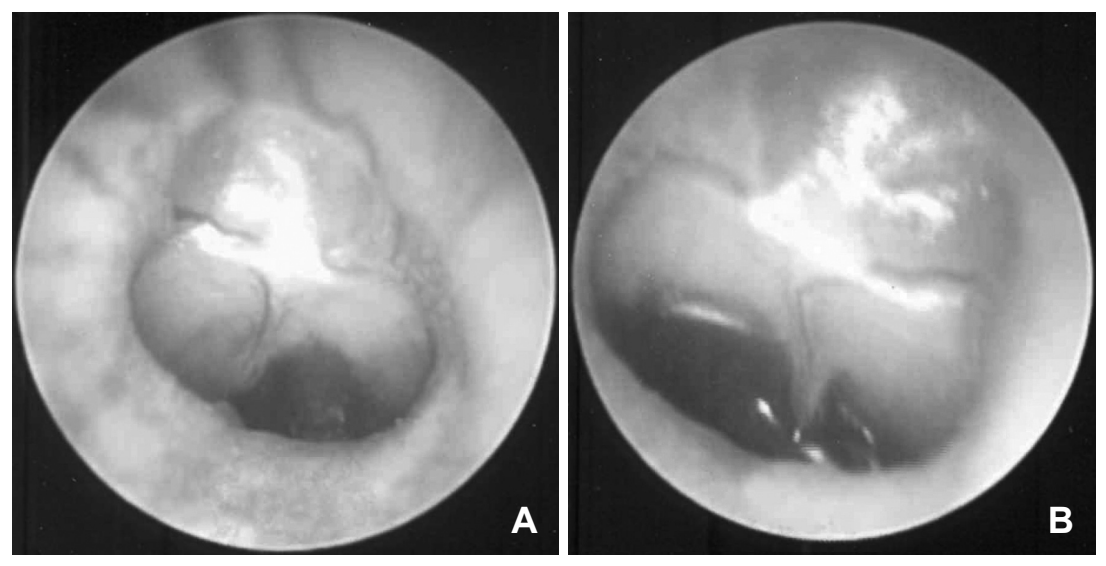

shown to induce otitis media with effusion in half of rats $24 \mathrm{~h}$ after injection. ${ }^{12)}$

Spontaneous otitis media with effusion was recently demonstrated in a mouse model of a specific genetic mutation in a G protein-coupled receptor encoded by the $\operatorname{Oxgrl}$ gene. $^{15)}$

\section{Animal Models of Acute Otitis Media}

Injection of bacteria through the tympanic membrane is the most common way of inducing acute otitis media in animals (Fig. 3). ${ }^{16)}$ Common causes of infections include Streptococcus pneumoniae, Haemophilus influenzae, and Moraxella catarrhalis. Methicillin-resistant Staphylococcus aureus and Pseudomonas aeruginosa are also commonly used in experimental studies. An insufficient inoculum does not induce acute otitis media, and an excess can result in meningitis or sepsis. Thus, bacteria should be diluted to the appropriate levels.

Systemic reactivation of otitis media with effusion in a rat model was studied using peptidoglycan-polysaccharide. ${ }^{17)} \mathrm{Ge}-$ netic predisposition has been demonstrated in animal models of middle ear inflammation. ${ }^{18)}$

\section{Animal Models of Cholesteatoma}

Four theories of cholesteatoma development exist: metaplasia, immigration, hyperplasia, and retraction pocket formation. The retraction pocket theory is the most widely used. Animal models of cholesteatoma are usually based one of these theories.

Several categories of animal models of cholesteatoma are available. ${ }^{7)}$ One involves a surgical method, such as external auditory canal ligation, Eustachian tube blocking, or autologous dermal implantation. The other involves the use of chemical materials in conjunction with various delivery methods.

The external auditory canal ligation model is a relatively simple and well-documented method ${ }^{19)}$; however, Mongolian gerbils are required. Mongolian gerbils are useful because cholesteatoma can be easily induced by ligation, and spontaneous cholesteatoma formation is known to occur. Cholesteatoma is induced in $100 \%$ of ligated ears; this is a very high success rate 


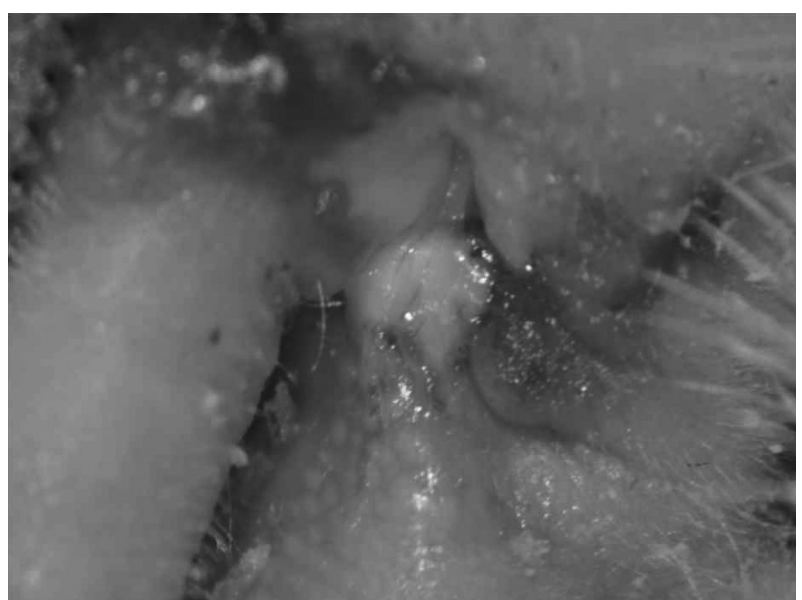

Fig. 4. Result 14 days after induction of cholesteatoma by injection of propylene glycol.

in terms of experimental studies of cholesteatoma. Unfortunately, Mongolian gerbils are very expensive in Korea.

Eustachian tube blocking was shown to induce cholesteatoma formation in three-quarters of Mongolian gerbils within 16 weeks. ${ }^{20)}$ Cholesteatoma can be induced by removing the pars flaccida of the tympanic membrane and transplanting a tympanic membrane to the defect of the original tympanic membrane. $^{21)}$

Chemical reagents are usually delivered by injection. Reagents can be injected through the tympanic membrane or bulla. Materials that can induce cholesteatoma include talcum powder, dimethylbenzanthracene, propylene glycol, and latex. $^{22,23)}$ Propylene glycol (concentration, 90\%) results in induction of cholesteatoma in most rats (Fig. 4).

\section{Conclusions}

Several animal models of otitis media are available. Unfortunately, none are ideal. Researchers who study otitis media should be familiar with the strengths and weak points of each model when selecting the model most appropriate for their purposes. Further physiological animal models of otitis media should be developed.

\section{REFERENCES}

1) Rovers MM. The burden of otitis media. Vaccine 2008;26 Suppl 7: G2-4.

2) Teele DW, Klein JO, Rosner B. Epidemiology of otitis media during the first seven years of life in children in greater Boston: a prospective, cohort study. J Infect Dis 1989;160:83-94.

3) Shekelle P, Takata G, Chan LS, Mangione-Smith R, Corley PM, Morphew T, et al. Diagnosis, natural history, and late effects of otitis media with effusion. Evid Rep Technol Assess (Summ) 2002:1-5.
4) Casselbrant ML, Mandel EM. Acute Otitis Media and Otitis Media with Effusion. In: Flint PW, Haughey BH, Lund VJ, Niparko JK, Richardson MA, Robbins KT, editors. Cummings OtolaryngologyHead and Neck Surgery. 5th ed. Philadelphia, PA: Mosby;2010. p.2761-77.

5) Capra AM, Lieu TA, Black SB, Shinefield HR, Martin KE, Klein JO. Costs of otitis media in a managed care population. Pediatr Infect Dis J 2000;19:354-5.

6) Proud GO. Eustachian tube function and middle ear pressures as they influence susceptibility to disease. Laryngoscope 1972;82: 1643-6.

7) Yamamoto-Fukuda T, Takahashi H, Koji T. Animal models of middle ear cholesteatoma. J Biomed Biotechnol 2011;2011:394241.

8) Preciado D, Kuo E, Ashktorab S, Manes P, Rose M. Cigarette smoke activates NFKB-mediated Tnf- $\alpha$ release from mouse middle ear cells. Laryngoscope 2010;120:2508-15.

9) DiFranza JR, Aligne CA, Weitzman M. Prenatal and postnatal environmental tobacco smoke exposure and children's health. Pediatrics 2004;113(4 Suppl):1007-15.

10) Proud GO, Odoi H. Effects of Eustachian tube ligation. Ann Otol Rhinol Laryngol 1970;79:30-2.

11) Piltcher OB, Swarts JD, Magnuson K, Alper CM, Doyle WJ, Hebda PA. A rat model of otitis media with effusion caused by eustachian tube obstruction with and without Streptococcus pneumoniae infection: methods and disease course. Otolaryngol Head Neck Surg 2002; 126:490-8.

12) Aynali G, Yariktaș M, Yasan H, Karahan N, Başpinar S, Tüz M, et al. The effects of methylprednisolone, montelukast and indomethacine in experimental otitis media with effusion. Int J Pediatr Otorhinolaryngol 2011;75:15-9.

13) Russell JD, Giles SJ. Persistent otitis media with effusion: a new experimental model. Laryngoscope 1998;108(8 Pt 1):1181-4.

14) Huang Q, Zhang Z, Zheng Y, Zheng Q, Chen S, Xu Y, et al. Hypoxia-inducible factor and vascular endothelial growth factor pathway for the study of hypoxia in a new model of otitis media with effusion. Audiol Neurootol 2012;17:349-56.

15) Kerschner JE, Hong W, Taylor SR, Kerschner JA, Khampang P, Wrege KC, et al. A novel model of spontaneous otitis media with effusion (OME) in the Oxgrl knock-out mouse. Int J Pediatr Otorhinolaryngol 2013;77:79-84.

16) Giebink GS. Otitis media: the chinchilla model. Microb Drug Resist 1999;5:57-72.

17) Jewett BS, Prazma JP, Hunter SE, Rose AS, Clark JM, Sartor BR, et al. Systemic reactivation of otitis media with effusion in a rat model. Otolaryngol Head Neck Surg 1999;121:7-12.

18) Clark JM, Brinson G, Newman MK, Jewett BS, Sartor BR, Prazma $\mathrm{J}$, et al. An animal model for the study of genetic predisposition in the pathogenesis of middle ear inflammation. Laryngoscope 2000;110: $1511-5$.

19) Kim HJ, Chole RA. Experimental models of aural cholesteatomas in Mongolian gerbils. Ann Otol Rhinol Laryngol 1998;107:129-34.

20) Wolfman DE, Chole RA. Experimental retraction pocket cholesteatoma. Ann Otol Rhinol Laryngol 1986;95(6 Pt 1):639-44.

21) Yamamoto-Fukuda T, Hishikawa Y, Shibata Y, Kobayashi T, Takahashi H, Koji T. Pathogenesis of middle ear cholesteatoma: a new model of experimentally induced cholesteatoma in Mongolian gerbils. Am J Pathol 2010;176:2602-6.

22) Massuda ET, Oliveira JA. A new experimental model of acquired cholesteatoma. Laryngoscope 2005;115:481-5.

23) White SJ, Wright CG, Robinson KS, Meyerhoff WL. Effect of topical hyaluronic acid on experimental cholesteatoma. Am J Otolaryngol 1995;16:312-8. 